Review

\title{
Robust Fiber-Optic Sensor Networks
}

\author{
Rosa Ana PEREZ-HERRERA*, Montserrat FERNANDEZ-VALLEJO, \\ and Manuel LOPEZ-AMO
}

\author{
Department of Electric and Electronic Engineering, Public University of Navarra, 31006 Pamplona, Spain \\ *Corresponding author: Rosa Ana PEREZ-HERRERA_E-mail: rosa.perez@unavarra.es
}

\begin{abstract}
The ability to operate despite failure will become increasingly important as the use of optical sensor networks grows, and the amount of sensing information to be handled by a sensor network is increasing, especially for safety and security applications. In this review, the four categories of protection to allow service to be reestablished after a failure (dedicated/shared and line/path) are thoroughly discussed. This paper also presents an overview of the most representative robust fiber-optic sensor systems, discussing their schemes, pros and cons.
\end{abstract}

Keywords: Self-healing, robust, resilience, fiber-optic sensor multiplexing, fiber Bragg gratings (FBGs), fiber-optic networks

Citation: Rosa Ana PEREZ-HERRERA, Montserrat FERNANDEZ-VALLEJO, and Manuel LOPEZ-AMO, "Robust Fiber-Optic Sensor Networks," Photonic Sensors, DOI: 10.1007/s13320-012-0083-2.

\section{Introduction}

Fiber-optical sensor networks, by and large, can be defined as a group of two or more fiber optic multiplexed sensors which are deployed either directly inside the element to be assessed or very close to it. The most fundamental motivation for multiplexing fiber optic sensors is the cost. Wavelength division multiplexing (WDM) is one of the best methods since it uses optical power efficiently, the enormous bandwidth available from the standard optical fibers can be employed in a competent way, and also, it is easily integrated into other multiplexing methods, allowing a large number of sensors in a single fiber line [1, 2]. In fact, the association of active fiber optic networks, which include optical amplification, with the WDM technology, has been the responsible for the revolution of fiber optic sensor networks [3, 4]. In addition, fiber-optical sensor networks present some advantages in comparison with the wireless network: vast bandwidth, electromagnetic interference is not an issue, data privacy is enhanced, and long unamplified transmission ranges are enabled. Furthermore, these networks offer the intrinsic benefits of the optical fiber, thus, they can be used in combustible, radioactive, or chemically corrosive environments, and even they can be imbedded within the structures [5-7]. Among the wide variety of available sensors, usually, fiber Bragg gratings (FBGs) are the strongest candidate for this kind of systems due to their numerous advantages. It is noteworthy the FBGs have high multiplexing capability and wavelength-encoded information, thereby, the information remains immune to power fluctuations $[8,9]$.

In this technological framework, fiber optical networks represent a significant improvement over

Received: 20 July 2012 / Revised version: 6 August 2012

(C) The Author(s) 2012. This article is published with open access at Springerlink.com 
traditional sensors networks, and consequently, they have emerged as a powerful tool for condition evaluation of the system under consideration [5]. They have found a promising niche in the field of structural health monitoring (SHM) which refers to the use of in-situ, continuous or regular measurement and analyses of key structural and environmental parameters under operating conditions, for the purpose of warning impending abnormal states or accidents at an early stage to avoid causality as well as giving the maintenance and rehabilitation advice [7]. Fiber-optic sensor networks provide sensing solutions for almost all kind of applications and environments: from large scale structures, including bridges and other civil constructions, to large natural environments $[1,6$, 10].

Despite the fact that many fiber optical networks for sensors multiplexing can be found in the literature [11-17], they still present two main challenges common to any optical network irrespective of whatever their final application. The first demand is the need to increase the number of sensors multiplexed on a single network while ensuring good signal quality. The second demand is to ensure service continuity in the event of point failure(s) on the network [18, 19], which will be the key issue for practical FBG sensor systems because it will enhance the reliability of FBG sensor systems $[18,20]$. As a matter of fact, the continued operation of the sensor network after accidental or malicious damage is of increasing importance when the structure being monitored is of high value (oil pipelines, power transmission lines, etc.); human safety is at risk (bridges, dams, chemical storage sites, nuclear plants, etc.) or perimeter security is a concern (airports, banks, etc.) [7, 21]. The use of optical switches has been demonstrated to be an ideal option to increase the number of multiplexed sensors in this kind of networks, as it has been shown in $[17,21]$.

From our point of view, the ability to operate despite failure will become increasingly important as the use of optical sensor networks grows, and the amount of sensing information to be handled by a sensor network is increasing, especially for these safety and security applications. Therefore, resilient fiber-optic networks are going to be thoroughly discussed in this review.

As aforementioned, robustness concept entails the ability of continuous operating despite one or more points of failure on the network [20]. Fibers can be broken by human activities or natural events, causing light-path interruption. When this fact happens to an interconnect fiber, only one sensor is isolated. However, failures in the main fibers, which are used to guide all the signals to reduce costs, render the network inoperative. Thus, the appropriate design of a robust optical fiber sensor network should satisfy three criteria simultaneously: firstly, the network must withstand at least one fiber failure at any point; secondly, the network must operate with nominally equal transmission losses for all sensing channels in passing from the transmitter mode to the receiver one, both in normal operation and after recovery from a failure; thirdly, it must be possible to signal the failure and to take the required actions without external resources such as dedicated fibers or radio links [20]. This third criterion which involves "signaling" is the most severe; it is responsible for sending messages to the networks management computers in order to warn of a failure and subsequently request appropriate remedial actions. Nevertheless, a detailed discussion of this point goes beyond the scope of this review because we concentrate on architectural principles. In addition, appropriate protection protocols also enable to detect which fiber segment has failed without the need for optical time domain reflectometry [19].

In the literature, only some articles explained the protection categories and signaling requirements to ensure service continuity $[19,22]$. There are, in general, four categories of protection to allow 
service to be reestablished after a failure: "dedicated" or "shared" protection, each of these has sub-categories called "path" and "line" protection, as a result, dedicated line, dedicated path, shared line and shared path are the four classes. All of them have direct counterparts in telecommunications networks [22].

From a hardware point of view, the difference between dedicated and shared protection, usually, comes from the way the sensor unit is joined to the network. Dedicated protection utilizes a coupler (see Fig. 1) while shared protection uses a switch (see Fig. 2). In dedicated protection, the signal travels through both working and protection fibers simultaneously. The receiver will accept only one, and the other will be discarded. The criterion of acceptance is usually by default of the signal provided by working fibers. Although the $1 \times 2$ couplers typically cause a minimum loss of $6 \mathrm{~dB}$, they are relatively low-cost components. In shared protection, the switch is chosen if the signal travels via working or protection fibers. Only the working fibers are used in normal operation, and only the protection fibers in the event of a cable fail to provide the required redundancy. The additional switch has low insertion losses but it is an expensive element which demands the power supply [21].

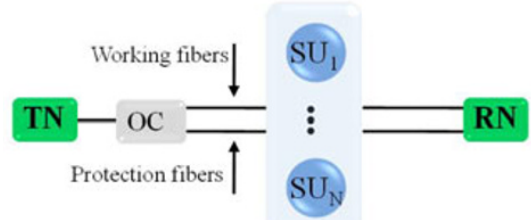

Fig. 1 Dedicated protection.

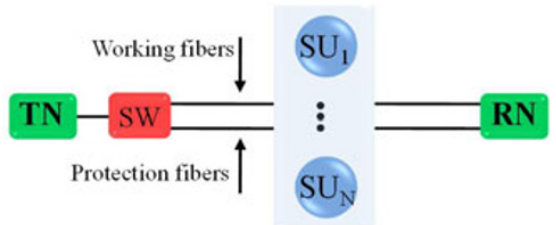

Fig. 2 Shared protection.

Making a comparison between both options: dedicated protection offers clear advantages from the point of view of cost, simplicity of automatic protection switching (APS) software, ease of signaling and the ability to withstand multiple failures in some topologies; while shared protection is more suitable when the losses are a limiting factor, and it is worth noticing its ability to offer spatial reuse $[19,21]$.

On the other hand, path protection and line protection differ in the form of protection. In path protection, each sensor is protected individually by the switch located in the transmission or receiver node which reroutes the information in the event of a failure in the network (see Fig. 3). However, in line protection, the sensors are protected by the nearest switches to the failure, and such switches do not belong to the transmission/receiver node but they are placed in the sensor network itself (see Fig.4) [23]. Thus, if there is a failure, the network is able to reconfigure the route. As it will be explained subsequently, line protection can be associated with self-healing capability. A hybrid dedicated line/path strategy can be a good possibility when a small group of sensors is particularly important, they can be protected using path protection, while the others can use line protection [24].

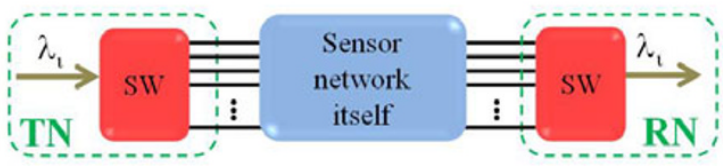

Fig. 3 Path protection

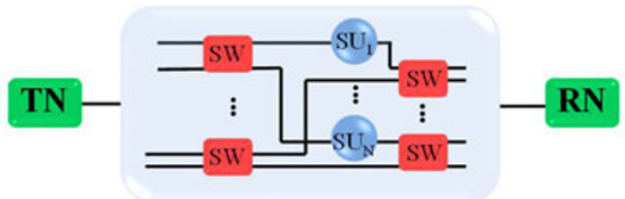

Fig. 4 Line protection.

Table 1 shows the definition of the different symbols used in the configurations.

The choice of a protection scheme is determined by the performance, cost and final application of the network. To give an idea of the different complexity of each protection system, one can take into account 
the number of switches. In an optical network with $N$ sensors, the amount of switches can vary notably depending on the kind of protection. On balance, as can be seen in Figs. 1 to 4, dedicated protection is preferable for most applications because the network uses entirely passive components. Consequently, the cost and failure probabilities are lower, no electrical power supplies are needed, and signaling is relatively simple. When dedicated protection is combined with path protection, the sensor network itself keeps the passive nature, thus the complexity is quite similar. However, in the case of using shared or line protection, the sensor network itself needs include switches. In particular, when line protection is used, the number of switches increases significantly. This aspect offers perhaps a more sophisticated robust capability but, on the other hand, raises the cost.

Table 1 Definition of the different symbols used in the configurations.

\begin{tabular}{cc}
\hline Symbol & Definition \\
\hline $\mathrm{CO}$ & Central office \\
$\mathrm{TN}$ & Transmission node \\
$\mathrm{RN}$ & Receiver node \\
$\mathrm{Mux}$ & Multiplexer \\
Demux & Demultiplexer \\
$\mathrm{W}_{\mathrm{i} / \mathrm{o}}$ & Input/output working fiber \\
$\mathrm{P}_{\mathrm{i} / \mathrm{o}}$ & Input/output protection fiber \\
$\mathrm{SU}$ & Sensor unit \\
$\mathrm{S}$ & Sensor \\
$\mathrm{OC}$ & Optical coupler \\
$\mathrm{SW}$ & Switch \\
$2 \mathrm{SW}$ & Node with 2 switches \\
$3 \mathrm{SW}$ & Node with 3 switches \\
\hline
\end{tabular}

Telecommunications service providers are well aware of the loss of revenue and customer confidence that follows accidental or malicious damage to transmission infrastructure. To this end, metropolitan and wide area networks are routinely configured as "self-healing rings" to perform "protection switching" in the event of a severed fiber. Many studies of survivable communication networks have been performed [25-27]. However, in comparison with their counterpart telecommunications networks, few studies have been published on multiplexed fiber sensor arrays that are designed to continue service in the event of unintended fiber damage [28-31].

Optical fiber sensor networks differ from their telecommunications counterparts in many ways that determine their design and operation. The sensors are preferably small, low cost and electrically passive. Fiber interconnects transport unmodulated waves to interrogate the sensors, where the measure imposes a modulation before passing to the receivers. Operation is analogue but the modulation rates are some orders of magnitude lower than that in communication, and thus slow switching speeds can be acceptable. In contrast, communication networks normally convey digital signals between large nodes, where electrical power supplies and optical amplification are available. Protection switching must normally be performed less than $50 \mathrm{~ms}$ to limit the data loss. Digital data frames or packets with overhead bytes for synchronization, error checking, signaling, protection switching and other vital management functions can be included. The synchronization needed for packet interleaving in time division multiple access can be ensured through digital ranging protocols [32]. However, in the absence of (costly) power feeds outside the end nodes, none of these facilities is usually available in sensor networks, precluding many of the network protection techniques used in communication. Although one can seek guidance from telecommunications practice, new approaches are required for analogue robust fiber sensor networks.

Table 2 summarizes the state of the art of resilient fiber-optic sensors systems in chronological order where the most important characteristics are pointed out. To the best of our knowledge, this is the first comprehensive evaluation of protection fiber optical networks which involves all proposals designed to date, both experimentally and theoretically, related to this topic. 
Table 2 State of the art of robust fiber-optic sensor systems.

\begin{tabular}{|c|c|c|c|}
\hline Year/Ref. & Network topology & $\begin{array}{l}\text { Experimental } \\
\text { results }\end{array}$ & Remarks \\
\hline $2002 /[33]$ & Ring fiber laser & Yes & Shared protection \\
\hline $2003 /[34]$ & Linear fiber laser & Yes & $\begin{array}{l}\text { Sensing units follow a star configuration and comprise a series of concatenated ring subnets } \\
\text { Shared protection }\end{array}$ \\
\hline $2003 /[35]$ & Linear fiber laser & Yes & $\begin{array}{l}\text { Sensing units with ring architecture } \\
\text { A depth discussion of the practical limitations of the scheme is included } \\
\text { Self-healing }\end{array}$ \\
\hline $2003 /[18]$ & Linear fiber laser & Yes & $\begin{array}{l}\text { Bidirectional ring topology for the FBG sensors } \\
\text { Self-healing }\end{array}$ \\
\hline $2004 /[28]$ & Linear fiber laser & Yes & $\begin{array}{l}\text { Ring topology for the FBG sensors } \\
\text { Self-healing }\end{array}$ \\
\hline $2007 /[36]$ & Double-ring & Yes & $\begin{array}{l}\text { Power transparency through Raman amplification } \\
\text { Dedicated protection }\end{array}$ \\
\hline 2007/[19] & Double ladder bus & No & Theoretical comparison of the four protection categories of a double ladder bus self-healing network \\
\hline $2007 /[21]$ & Double ladder bus & No & Theoretical study of the four protection categories in different double ladder bus resilient networks \\
\hline $2008 /[37]$ & Liner fiber laser & Yes & $\begin{array}{l}\text { Multi-ring architecture for the FBG sensors } \\
\text { Shared protection }\end{array}$ \\
\hline $2009 /[23]$ & $\begin{array}{l}\text { Symmetrical dual } \\
\text { fiber bus }\end{array}$ & No & $\begin{array}{l}\text { Theoretical study of the optical amplified fiber network with "dedicated-line" or "dedicated-path" } \\
\text { The network can support any kind of sensors }\end{array}$ \\
\hline $2009 /[38]$ & Double-ring & Yes & $\begin{array}{l}\text { Power transparency through Raman amplification } \\
\text { Share protection } \\
\text { The network can support any kind of sensors }\end{array}$ \\
\hline $2009 /[39]$ & Linear fiber laser & Yes & $\begin{array}{l}\text { Active components are not required in the sensor units } \\
\text { Enhanced version of [33] with multi-ring architecture for the FBG sensors } \\
\text { Shared protection }\end{array}$ \\
\hline $2009 /[40]$ & Star & No & $\begin{array}{l}\text { FBG arrays as ring sensing subnets } \\
\text { Two self-healing architectures are proposed: both based on shared protection }\end{array}$ \\
\hline $2009 /[41]$ & Ring fiber laser & Yes & $\begin{array}{l}\text { Two level-ring architecture } \\
\text { Shared line protection }\end{array}$ \\
\hline $2010 /[31]$ & Linear fiber laser & Yes & $\begin{array}{l}\text { Combination of WDM and TDM } \\
\text { FBGs sensors in star-ring topology } \\
\text { Self-healing }\end{array}$ \\
\hline $2010 /[42]$ & Linear fiber laser & Yes & $\begin{array}{l}\text { Long-distance }(50 \mathrm{~km}) \text { sensor system using a multiwavelength Raman laser } \\
\text { Shared protection }\end{array}$ \\
\hline $2010 /[43,44]$ & Ring fiber laser & No & $\begin{array}{l}\text { Three dimensional mesh-based } \\
\text { Sensing system }\end{array}$ \\
\hline $2011 /[45]$ & Linear fiber laser & Yes & $\begin{array}{l}\text { Ring topology for the sensors } \\
\text { Shared protection }\end{array}$ \\
\hline $2011 /[20]$ & Double ladder bus & No & $\begin{array}{l}\text { Mathematical model of a WDM resilient optic sensor network to multiplex optical sensors with dedicated line } \\
\text { protection }\end{array}$ \\
\hline $2011 /[29]$ & Ring fiber laser & Yes & $\begin{array}{l}\text { Tunable EDF laser with } 25-\mathrm{km} \text { cavity length } \\
\text { Shared protection system }\end{array}$ \\
\hline $2011 /[30]$ & Linear fiber laser & Yes & $\begin{array}{l}\text { FBG sensors in a delta-ring configuration } \\
\text { Self-healing }\end{array}$ \\
\hline $2012 /[46]$ & $\begin{array}{l}\text { WDM mesh } \\
\text { network }\end{array}$ & Yes & Optical add-drop multiplexer for WDM mesh networks \\
\hline
\end{tabular}




\section{Robust fiber-optic sensor systems}

This section is devoted to explain more carefully the most representative robust fiber-optic sensor systems presented in Table 2, discussing their schemes, pros and cons. They are going to be evaluated not in chronological order as in the list, but taking into account the system topology.

There have been many studies of how to ensure survivability of telecommunications fiber networks and sensor radio networks. However, to date there have been few proposals for survivable fiber networks for sensors. In the following subsections, we present a classification of the robust fiber-optic sensor topologies commonly used for this purpose, along a sampling of the results from recent research in this area.

\subsection{Ring networks}

One of the most typical topologies is the optical ring network, because of that, the survivability and resilience of this type of topologies when a fault occurs are going to be examined in detail.

As it was previously said, providing high survivability to a fiber network for sensors will become increasingly important for safety-critical applications and where the sensors monitor high value infrastructure [47]. For different applications, the survivability of the network may be of total necessity, and for other applications it is acceptable to sacrifice a part of the network but continue with the remainder operable all the time. If a sensor node fail within a network, it may be impossible to repair it because it is situated in a place that is not meant to be reached and mended. That is why it is so important to have the rest of the network operational despite of such a failure. We may find it acceptable to lose a node in the network and consider it still operational, but to lose the whole network because a single node means that the network has no survivability and is considered to be unreliable.

The dual-ring topology in combination with
WDM, synchronous digital hierarchy (SDH), or synchronous optical networking (SONET) offers great flexibility and the ability to function as a self-healing ring that is able to restore the service by itself within tens of milliseconds after an accident has occurred [47]. Self-healing rings are able to completely restore lost traffic in case a cable is cut somewhere in the network. Nowadays, self-healing rings are preferred as a topology in wide area networks (WANs), metropolitan area networks (MANs) and sub-sea communication, where the need for good protection against failures is of great importance. Dual-rings are differentiated depending on the way that traffic is utilized among the nodes that are used in SDH networks.

There are two types of self-healing rings: unidirectional path-switched ring (UPSR) and bidirectional line-switched ring (BLSR). BLSRs are not that interesting because the components necessary for the appropriate operation of the network greatly increase costs and are not required for the purpose of sensing. Furthermore, they require more demanding control software. The working and protection cables are situated away from each other as much as possible in order to reduce the chance of a fault affecting both fibers at the same time. This is an essential requirement in order to have successful resilience using a double ring.

Moreover, the sub-network connection protection (SNCP) ring is a dedicated protection architecture [47]. The nodes are connected in a ring configuration with one fiber pair connecting adjacent nodes, as shown in Fig. 5. One fiber on a link is used as the working fiber, and the other one is dedicated for protection.

This double ring construction allows simultaneous interrogation sensors from both rings, which confers two advantages. Firstly, one could obtain two measurements of each sensor and thereby increase the precision. Secondly, there is sufficient redundancy to provide resilience against fiber failure. Resilience to accidental fiber damage in sensors 
networks has been proposed [48], and it will become more important with the increased need to monitor valuable or safety-critical structures.

Both rings in this type of networks incorporate passive directional couplers to connect the sensors. Where necessary, they allow individual sensors to be changed (e.g. to a different wavelength) or eliminated completely without disturbing the transmission fiber of the rings. They also avoid laser oscillation and associated instabilities that might occur if WDM couplers are used in an amplified network. Thus, the topology shown in Fig. 5 is compatible with the use of optical amplification to allow the number of nodes (taps) in the ring to be increased, while maintaining the power levels of the signals exiting the sensors [36].

The resilience of the network is offered through what is known in telecommunications engineering as "dedicated protection" (also called " $1+1$ protection") [49]. Signals are launched on to both rings from the transmitters. Two nominally identical signals arrive at the receivers from each sensor. These two signals may be used to average signal and noise levels in normal operation, having an improved measurement, or the one with the higher optical signal to noise ratio (OSNR) may be selected. When a fiber failure occurs, one of the signals is interrupted, and the receiver automatically switches to the one that survives (see Fig. 6). Dedicated protection is a relatively low complexity means of providing resilience because, in the network of Fig. 5, it does not require telemetry signaling to communicate the need for protection switching between the various parts of the network, and this greatly simplifies any control software.

When a fault occurs (node breakdown or cable cut), the network remains operational, but the determination of the fault is an issue. Using optical time-domain reflectometry (OTDR) helps us to approximate the location of the fault by using pulses of light that propagate along the network and return echoes from places in which the refractive index of the fiber changes (usually where the cable is cut).

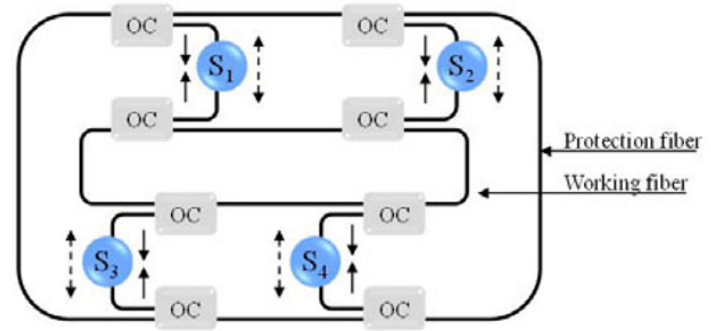

Fig. 5 Double ring multiplexing network (solid: input signal; dashed: reflected signal).

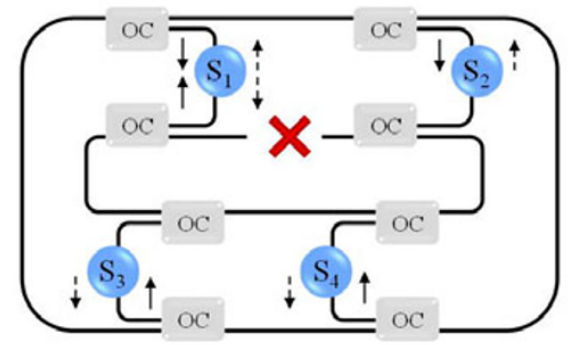

Fig. 6 Double ring multiplexing network with a fiber failure.

This topology has been used to develop several double ring amplified networks using Raman amplification for WDM of FBG sensors such as in $[36,38]$. Their key attribute is to enable service continuity after a failure in one of their constituent fibers. The first configuration (see Fig. 7) used FBGs for both the sensing function and the unique identification of the sensors, according to the wavelengths reflected. The second configuration (see Fig. 8) used separate sensors based on fused optical fibers but the FBGs continued to allow their unique identification. Resilience was provided by dedicated and shared protection in the first and second network designs, respectively. The first design was relatively simple to implement, and the second one could operate with many sensor types for various measurements. It has been used a combination of discrete and distributed Raman amplification to compensate for the losses imposed by the optical taps in the networks, achieving power transparency with total pump power of $1.2 \mathrm{~W}$ and $0.8 \mathrm{~W}$ for the first and second ring designs, respectively. The obtained results therefore indicated the scalability of the networks to serve greater 
numbers of sensors.

A number of novel topologies, which enhanced the reliability and survivability in the long-reach fiber distance, have been also proposed and experimentally investigated. In [29], the sensing mechanism was based on an erbium-doped fiber (EDF) ring laser with a cavity length of $25 \mathrm{~m}$ for detecting the multiple FBG sensors in the network. One of the main advantages of this proposed fiber laser scheme is that the long distance sensing systems can be integrated in the fiber access network to reduce the cost of sensor infrastructure in the future.

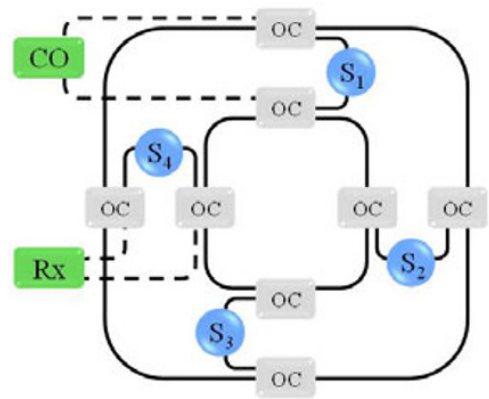

Fig. 7 Experimental setup for the first double ring amplifier adapted from [38].

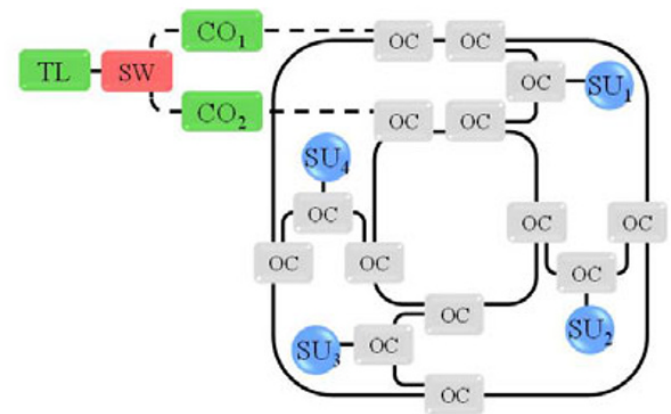

Fig. 8 Experimental setup for the second double ring amplifier adapted from [38].

\subsection{Double ladder bus}

Another option to design wavelength multiplexed fiber sensor networks that can withstand one or more cable failures is the double ladder bus. Bus structures have been widely used for frequency division multiplexing (FDM), time division multiplexing (TDM), and coherence multiplexing
[50]. However, fiber Bragg gratings, which are now widely available low-cost and low-loss wavelength reflectors, have enabled WDM techniques to increase the importance among their competitors. As it is well known, the gratings can act as sensors themselves $[50,51]$ or can be used to identify the sensors within the network $[52,53]$. In this way, WDM bus networks make efficient use of the fiber to enable the multiplexing of intensity or interferometric sensors that respond to many measurements. Therefore, they are not application-specific, which confers cost advantage.

As it was previously pointed out, there are four categories of protection - dedicated line, dedicated path, shared line and shared path - all have particular characteristics, determining the locations and numbers of failures against which they can protect their signaling requirements. The critical factors that determine the decision between these options are the cost, the complexity of the APS software, the ease of signaling, the ability to withstand multiple failures, the optical transmission impairments and the need for spatial reuse. These protection categories of double ladder bus self-healing networks have been theoretically analyzed [19, 22]. All these criteria are very demanding but they can be satisfied with appropriate network architectures. An extension of this work [19] but concentrated on the network amplification strategy has been also reported [23]. In this study, the power and amplified spontaneous emission (ASE) of a line-protected network to serve 40 sensors on the scale of a campus were simulated. Amplification was determined by remotely pumped spans of an erbium-doped fiber, and in this way, a crucial network attribute was demonstrated: operation was possible without electrical powering outside the end nodes. Moreover, several multiplexing networks, which included a remote power by light fiber optic switches, have been recently carried out.

One of the most satisfactory topologies is the "direct unidirectional sensor array", depicted in Fig.9. 
It has a relatively simple cabling configuration, which reduces the construction costs and fiber damage probabilities. There are two infrastructures connected to each sensor unit, described as "working" and "protection". The working fibers and cables are used in normal operation, and the protection fibers and cables provide the required redundancy. The working cable contains a distribution and an aggregation fiber, and they both have broadband couplers to connect to each sensor unit (SU) via the "working interconnect" fibers.

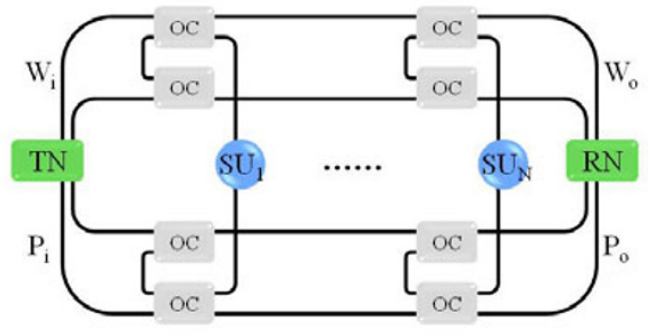

Fig. 9 Direct unidirectional sensor array network.

On the other hand, a mathematical model for these WDM self-healing optical fiber buses has been recently developed [20]. Here, it is shown that these networks can be also "self-diagnostic", which is defined to mean that it can determine failed element(s) from network management information, without requiring external resources. In that case, the information is the combination of channels that arrive at the reception points, and it permits unambiguous identification of all important element failures resulting from a single or double destruction event. This model is a matrix-vector formalism that divides the network into blocks and predicts which wavelengths arrive at the receiver node when damage has occurred in one or more places. All values of the power are discrete, being 1 or 0 , to indicate channel presence or absence, respectively. Similarly, a coefficient with the value of 1 or 0 is assigned to each network element, according to whether it is functional or has failed. A matrix-vector equation has been derived to predict the network connectivity of the propagating wavelengths. So, by monitoring all combinations of channel launch points and reception points in the network's end nodes, the failed network elements can be unambiguously identified from the received wavelengths. Single [20] and multiple failures within one or different blocks [54], categorizing and tabulating all combinations of surviving wavelengths, have been studied in detail.

Lastly, a number of experimental studies of its viability by providing evidence in favor of the model have been published. In these works, a representative sample of single or double failure sites has been tested, demonstrating the patterns of channel arrivals predicted by theory.

\subsection{Linear fiber lasers}

The last option to develop optical fiber sensor systems is based on fiber lasers structures which enhance the performance of the system; in particular, they offer the improved signal to noise ratio (SNR) when they are compared with nonlasing networks due to the fact that the noise associated with the amplification is employed in the lasing process. Thus, they are able to cope with the losses associated with the required devices that ensure the survivability of the robust system. Usually, these systems are based on linear fiber lasers [18, 28, 34, $35]$ or ring fiber lasers [30, 31]. But, it is important to take into consideration not only the topology of the whole system but also the allocation of the sensors and how the system works in order to have robustness capability.

In the literature, different topologies have been proposed to distribute the sensors: ring [18, 35], star-bus [28], delta-star [30], star-ring bus [31], multiring passive architecture [37, 39], and parallel distribution [42].

The first four topologies, shown in Fig. 10, have a key characteristic in common: all of them are self-healing architectures which means that they have reconfigurable capability to prevent the sensor networks from a fiber failure(s) which is supported by optical switches. In normal operation, all switches are in the default position, and the sensing 
signal has a fix path. However, in protection operation, when a fiber curt occurs, some switches change their launch point, thus the lost sensing information can be retransmitted under the new link reconstructed.

As can be seen in $[18,28,30,31,35]$ in protection operation, when the signal path has been modified, some sensors are interrogated from the opposite direction with respect to the direction in normal operation. This aspect can restrict the practical applicability of these self-healing architectures because of the fact that bidirectional sensors, such as FBGs, are the only sensors that can be used.

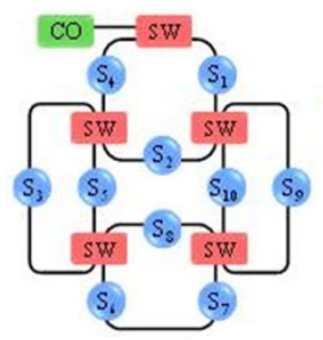

(a)

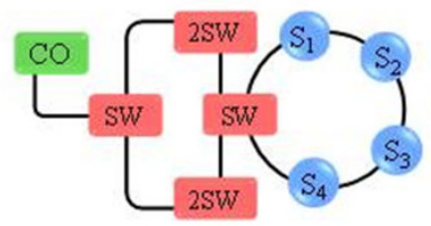

(b)

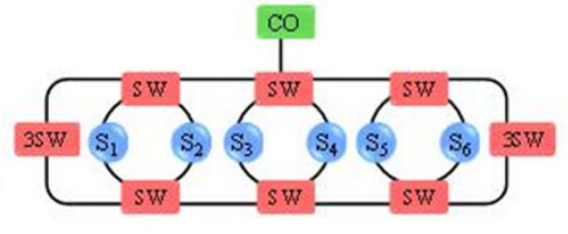

(c)

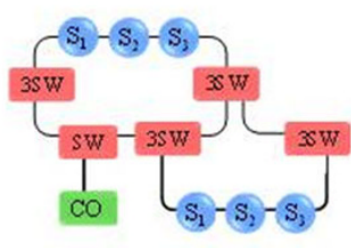

(d)

Fig. 10 Topologies to distribute the sensors: (a) ring, adapted from [18], (b) star-bus, adapted from [28], (c) delta-star, adapted from [30], and (d) star-ring bus architecture, adapted from [31].

It is evident that a pivotal device of the self-healing sensor networks is the switch. As aforementioned, it allows reconfiguring the signal path to ensure service continuity in the event of a point failure. However, switches are expensive devices which can increase considerably the cost of the system because of the fact that some networks need a big number of them. Switches also require the power supply; thus, these kinds of protection schemes are not suitable for certain application such as the case of sensor networks with remote sensing capability [55].

But it seems important to highlight that self-healing sensor networks, in terms of scalability and reliability, are promising candidates for the large scale multipoint FBG sensor network.

Architectures proposed in $[39,42]$ were passive sensing networks in the sense that they did not require active components in the network as could be seen in Fig. 11. It was obvious that the complexity of the network was reduced considerably and thereby also the cost. But, these aspects had a direct influence in the way of assuring robustness which was simpler than that in the case of self-healing concept. Figure 12 shows the obtained

results from the setup shown in Fig. 11(b) when one of the FBGs that acted as a sensor was placed into a climatic chamber in order to observe its behavior when temperature changed. It is possible to observe that the center wavelength shift presents a clear linear behavior [see Fig. 12(b)].

In both systems, a switch was used in order to make the system inherently resilient to fiber failures.

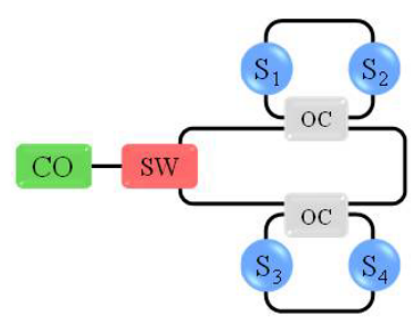

(a)

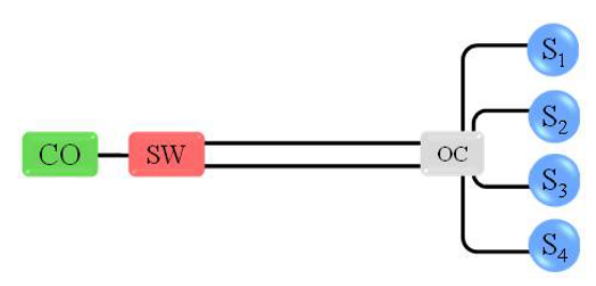

(b)

Fig. 11 Robust networks with (a) multiring passive architecture, adapted from [37, 39] and (b) sensors in parallel configuration, adapted from [42]. 
The $1 \times 2$ switch was located at the beginning of the network to perform the necessary selection of the launch point. With this goal, the systems used "dedicated protection" to re-establish service after a failure. In [37, 39], when the system had a fiber cut, the switch would choose the second launch point. Because of the design of the system, it could be easy to infer the location of the failure. In the particular case of [42], when the system worked in normal operation, the switch was connected to the "working fiber" but when a failure occurred, it was switched to the "protection fiber" (the other 50-km SMF, single mode fiber, was placed in parallel). Consequently, only the working fiber was used in normal operation, and the protection fiber was

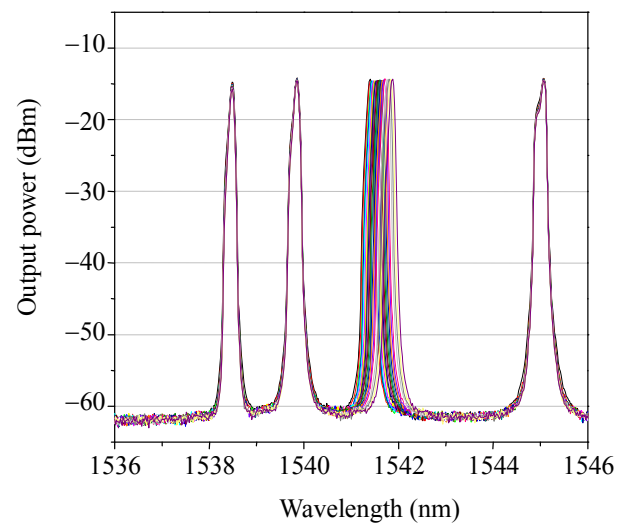

(a) activated in the event of a failure.

These kinds of robust networks with active elements only in the monitoring station can be a perfect choice for remote sensing applications where the power supply is not available in the sensing unit.

Consequently, the choice between self-healing topologies $[18,28,30,31,35]$ or simpler architectures with, for example, dedicated protection $[37,39,42]$ depends on the final applications which will establish the final design of the network and the real relevance of each sensor, and certain sensors can need the higher level of protection because of the measured parameter or because of the key position of the sensor.

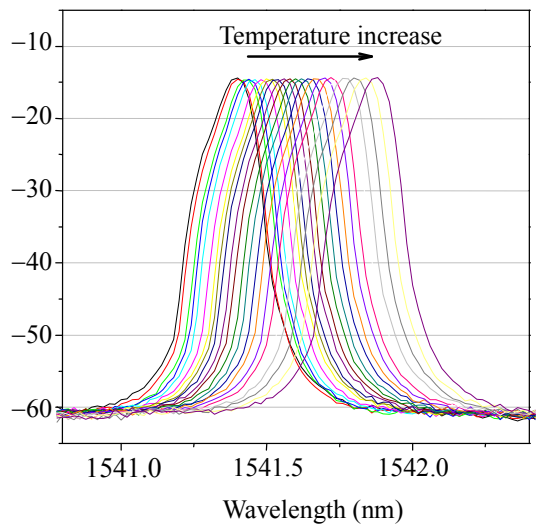

(b)

Fig. 12 Measured output spectrum of the resilient long-distance remote sensing system [Fig. 11(b)] when the FBG centered at $1541.4 \mathrm{~nm}$ was thermally stressed from $27{ }^{\circ} \mathrm{C}$ to $80{ }^{\circ} \mathrm{C}$ (a) with the detail of this measurement (b).

\subsection{Mesh sensing systems}

Although most of optical sensor networks are based on linear topologies, it is worth mentioning that bus, ring and star topologies have been also adopted in multipoint sensing systems to overcome system collapse resulting from breakpoints in the sensing system. However, not all kinds of breakpoints can be healed, and the sensing area is always limited in one dimension. In order to overcome this drawback, a number of mesh sensing systems to support more comprehensive sensing areas have been recently proposed and experimentally demonstrated [43, 44]. Furthermore, a number of novel optical add-drop multiplexers (OADMs) for WDM mesh networks have been also investigated [46]. In these topologies, based on the characteristics of a thin-film filter and an optical switch, the optical signal blocked by single or multiple fiber link failures can be reconnected through logical backup pathways.

In these topologies, the symmetric architecture ensures that the proposed sensing system can be accessed from any point. In such a configuration, the self-healing functionality can be applied to the whole sensing system by controlling the switching 
states of the optical switches to provide adequate sensing paths. Since the proposed architectures are almost always repeated and periodic, a three-dimensional sensing system, as shown in Fig. 13, can be realized by properly connecting all the peripheral edge switches. So, with such a sensing system, the self-healing function can be applied to the whole sensing system even for failure occurring at the nodes. The survivability of the sensing system has been enhanced, and the sensing elements could be distributed more densely.

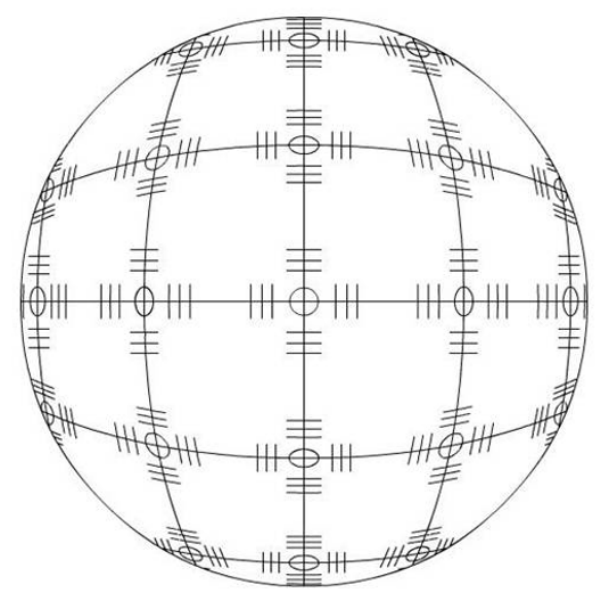

Fig. 13 Proposed three-dimensional mesh-based sensing system, adapted from [43].

\section{Conclusions}

This review presents an overview of the most representative robust fiber-optic sensor systems, discussing their topologies, pros and cons, along a sampling of the results from recent research in this area. There are several types of protection to allow service to be reestablished after a failure, and they can be classified as dedicated line, dedicated path, shared line or shared path. In this paper, these categories of protection have been discussed in detail.

Most robust fiber-optic sensor systems have been predesigned for protection against single failures. However, a number of novel topologies with the ability to withstand multiple failures, as well as a mathematical model for these WDM self-healing optical fiber buses, have been recently proposed.

We believe that survivability schemes for channel failures in fiber-optic sensors networks will become an important topic of research in the future, and, to the best of our knowledge, this is the first comprehensive evaluation of protection fiber-optics sensor networks, which involves a number of proposals designed to date, both experimentally and theoretically, related to this topic.

\section{Acknowledgment}

The authors are grateful to the Spanish Government project TEC2010-20224-C02-01. The authors acknowledge the useful discussions on this matter with Dr. Paul Urquhart.

Open Access This article is distributed under the terms of the Creative Commons Attribution License which permits any use, distribution, and reproduction in any medium, provided the original author(s) and source are credited.

\section{References}

[1] J. M. Lopez-Higuera, Handbook of optical fiber sensing technology, chapter 21, passive fiber optic sensor networks. England: John Wiley \& Sons Ltd., 2002, pp. 433-448.

[2] S. Yin, P. B. Ruffin, and F. T. S. Yu, Fiber optic sensors, chapter 1, overview of fiber optic sensors. Boca Raton, FL: CRC Press Taylor \& Francis Group, 2008, pp. 1-34.

[3] S. Diaz, S. Abad, and M. Lopez-Amo, "Fiber-optic sensor active networking with distributed erbium-doped fiber and Raman amplification," Laser and Photonics Reviews, vol. 2, no. 6, pp. 480-497, 2008.

[4] M. Lopez-Amo and J. M. Lopez-Higuera, Fiber Bragg gratings sensors: recent advancements, industrial applications and market exploitation, chapter 6, multiplexing techniques for FBG sensors. Bussum, The Netherlands: Bentham Science Publishers, 2011.

[5] J. L. Santos, O. Frazão, J. M. Baptista, P. A. S. Jorge, 
I. Dias, F. M. Araújo, et al., "Optical fiber sensing networks," in SBMO/IEEE MTT-S International Microwave and Optoelectronics Conference Proceedings, IMOC 2009, Belem, Brazil, Nov. 3-6, pp. 290-298, 2009.

[6] M. Majumder, T. K. Gangopadhyay, A. K. Chakraborty, K. Dasgupta, and D. K. Bhattacharya, "Fiber Bragg gratings in structural health monitoring-Present status and applications," Sensors Actuators A: Physical, vol. 147, no. 1, pp. 150-164, 2008.

[7] H. Li, D. Li, and G. Song, "Recent applications of fiber optic sensors to health monitoring in civil engineering," Engineering Structures, vol. 26, no. 11, pp. 1647-1657, 2004.

[8] A. D. Kersey, M. A. Davis, H. J. Patrick, M. LeBlanc, K. P. Koo, C. G. Askins, et al., "Fiber grating sensors," Journal Lightwave Technology, vol. 15, no. 8, pp. 1442-1462, 1997.

[9] K. O. Hill and G. Meltz, "Fiber Bragg grating technology fundamentals and overview," Journal Lightwave Technology, vol. 15, no. 8, pp. 1263-1276, 1997.

[10] S. Yin, P. B. Ruffin, and F. T. S. Yu, Fiber optic sensors, chapter 10, applications of fiber optic sensors. Boca Raton, FL: CRC Press Taylor \& Francis Group, 2008, pp. 397-434.

[11] M. Fernandez-Vallejo, S. Rota-Rodrigo, and M. Lopez-Amo, "Remote $(250 \mathrm{~km})$ fiber Bragg grating multiplexing system," Sensors, vol. 11, no. 9, pp. 8711-8720, 2011

[12] D. Leandro, A. Ullan, M. Lopez-Amo, J. M. Lopez-Higuera, and A. Loayssa, "Remote $(155 \mathrm{~km})$ fiber Bragg grating interrogation technique combining Raman, Brillouin and erbium gain in a fiber laser," IEEE Photonic Technology Letters, vol. 23, no. 10, pp. 621-623, 2011.

[13] A. Zornoza, R. A. Pérez-Herrera, C. Elosúa, S. Diaz, C. Bariain, A. Loayssa, et al., "Long-range hybrid network with point and distributed Brillouin sensors using Raman amplification," Optics Express, vol. 18, no. 9, pp. 9531-9541, 2010.

[14] T. Saitoh, K. Nakamura, Y. Takahashi, H. Iida, Y. Iki, and K. Miyagi, "Ultra-long-distance $(230 \mathrm{~km})$ FBG sensor system," in Proc. SPIE, vol. 7004, pp. 70046C-1-70046C-4, 2008.

[15] S. Diaz, G. Lasheras, and M. Lopez-Amo, "WDM bi-directional transmission over $35 \mathrm{~km}$ amplified fiber-optic bus network using Raman amplification for optical sensors," Optics Express, vol. 13, no. 24, pp. 9666-9671, 2005.
[16] M. J. F. Digonnet, B. J. Vakoc, C. W. Hodgson, and G. S. Kino, "Acoustic fiber sensor arrays," in Proc. SPIE (The International Society for Optical Engineering), vol. 5502, pp. 39-50, 2004.

[17] R. T. Chen, M. R. Wang, and T. Jannson, "Multiple-mode reconfigurable electro-optic switching network for optical fiber sensor array," in Proc. SPIE (The International Society for Optical Engineering), San Jose, CA, USA, Sep. 17, vol. 1374, pp. 223-236, 1990.

[18] P. Peng and S. Chi, "A reliable architecture for FBG sensor systems," Microwave Optics Technology Letters, vol. 39, no. 6, pp. 479-482, 2003.

[19] E. L. Izquierdo, P. Urquhart, and M. López-Amo, "Optical fiber bus protection architecture for the networking of sensors," in 2007 IEEE International Symposium on Intelligent Signal Processing, WISP, Alcala de Henares, Oct. 3-5, pp. 1-6, 2007.

[20] P. Urquhart, H. Palezi, and P. Jardin, "Optical fiber bus protection network to multiplex sensors: Self-diagnostic operation," Journal of Lightwave Technology, vol. 29, no. 10, pp. 1427-1436, 2011.

[21] C. W. Hodgson, M. J. F. Digonnet, and H. J. Shaw, "Large-scale interferometric fiber sensor arrays incorporating multiple optical switches," Optical Fiber Technology, vol. 4, no. 3, pp. 316-327, 1998.

[22] E. L. Izquierdo, P. Urquhart, and M. Lopez-Amo, "Protection architectures for WDM optical fiber bus sensor arrays," Journal of Engineering, vol. 1, no. 2, pp. 1-18, 2007.

[23] S. Ramamurthy, L. Sahasrabuddhe, and B. Mukherjee, "Survivable WDM mesh networks," Journal of Lightwave Technology, vol. 21, no. 4, pp. 870-883, 2003.

[24] O. G. López, K. Schires, P. Urquhart, N. Gueyne, and B. Duhamel, "Optical fiber bus protection network to multiplex sensors: amplification by remotely pumped EDFAs," IEEE Transactions on Instrumentation and Measurement, vol. 58, no. 9, pp. 2945-2951, 2009.

[25] D. Y. Zhou and S. Subramaniam, "Survivability in optical networks," IEEE Network, vol. 14, no. 6, pp. 16-23, 2000.

[26] A. A. M. Saleh and J. M. Simmons, "Architectural principles of optical regional and metropolitan access networks," Journal of Lightwave Technology, vol. 17, no. 12, pp. 2431-2448, 1999.

[27] X. Sun and P. Wei, "Using new models to enhance optical-fiber-sensor networks," SPIE Newsroom, Feb. 8, 2007, DOI: 10.1117/2.1200701.0522. 
[28] P. Peng, W. Lin, and S. Chi, "A self-healing architecture for fiber Bragg grating sensor network," in Proceedings of IEEE Sensors, vol. 1, pp. 60-63, 2004.

[29] C. Yeh, C. Chow, P. Wu, and F. Tseng, "A simple fiber Bragg grating-based sensor network architecture with self-protecting and monitoring functions," Sensors, vol. 11, no. 2, pp. 1375-1382, 2011.

[30] S. Kuo, P. Peng, J. Sun, and M. Kao, "A delta-star-based multipoint fiber Bragg grating sensor network," IEEE Sensors Journal, vol. 11, no. 4, pp. 875-881, 2011.

[31] P. Peng, J. Wang, and K. Huang, "Reliable fiber sensor system with star-ring-bus architecture," Sensors, vol. 10, no. 5, pp. 4194-4205, 2010.

[32] N. Miki and K. Kumozaki, Passive optical networks: principles and practice, chapter 5, ranging and dynamic bandwidth allocation. London, UK: Academic Press, Elsevier, 2007.

[33] P. Peng, H. Tseng, and S. Chi, "Self-healing fiber grating sensor system using tunable multiport fiber laser scheme for intensity and wavelength division multiplexing," Electronics Letters, vol. 38, no. 24, pp. 1510-1512, 2002.

[34] P. Peng, H. Tseng, and S. Chi, "A hybrid star-ring architecture for fiber Bragg grating sensor system," IEEE Photonics Technology Letters, vol. 15, no. 9, pp. 1270-1272, 2003.

[35] P. Peng, H. Tseng, and S. Chi, "A novel fiber-laser-based sensor network with self-healing function," IEEE Photonics Technology Letters, vol. 15, no. 2, pp. 275-277, 2003.

[36] R. A. Perez-Herrera, S. Diaz, P. Urquhart, and M. Lopez-Amo, "A resilient Raman amplified double ring network for multiplexing fiber Bragg grating sensors," in Proc. SPIE (The International Society for Optical Engineering), vol. 6619, pp. 66193E, 2007.

[37] C. H. Wang, C. H. Yeh, F. Y. Shih, C. W. Chow, K. C. Hsu, Y. Lai, et al., "Self-protection multi-ring-architecture fiber sensing system," Advanced Materials Research, vol. 47-50, pp. 793-796, 2008.

[38] M. Fernandez-Vallejo, R. A. Perez-Herrera, C. Elosua, S. Diaz, P. Urquhart, C. Bariáin, et al., "Resilient amplified double-ring optical networks to multiplex optical fiber sensors," Journal of Lightwave Technology, vol. 27, no. 10, pp. 1301-1306, 2009.

[39] C. Yeh, C. Chow, C. Wang, F. Shih, Y. Wu, and
S. Chi, "A simple self-restored fiber Bragg grating (FBG)-based passive sensing ring network," Measurement Science and Technology, vol. 20, no. 4, 043001-1-043001-5, 2009.

[40] H. Zhang, S. Wang, G. Wen, W. Ye, X. Chen, D. Jia, et al., "Large-scale self-healing architectures for fiber Bragg grating sensor network," in 9th International Conference on Optical Communications and Networks, ICOCN 2010, Nanjing, China, Oct. 24-27, pp. 99-102, 2010.

[41] P. Peng and K. Huang, "Fiber Bragg grating sensor system with two-level ring architecture," IEEE Sensors Journal, vol. 9, no. 4, pp. 309-313, 2009.

[42] M. Fernandez-Vallejo, S. Díaz, R. A. Perez-Herrera, D. Passaro, S. Selleri, M. A. Quintela, et al., "Resilient long-distance sensor system using a multiwavelength Raman laser," Measurement Science and Technology, vol. 21, no. 9, pp. 094017-1-094017-5, 2010.

[43] C. Y. Wu, K. M. Feng, P. C. Peng, and C. Y. Lin, "Three-dimensional mesh-based multipoint sensing system with self-healing functionality," IEEE Photonics Technology Letters, vol. 22, no. 8, pp. 565-567, 2010.

[44] C. Wu, F. Kuo, K. Feng, and P. Peng, "Ring topology based mesh sensing system with self-healing function using FBGs and AWG," in 2010 Conference on Optical Fiber Communication, Collocated National Fiber Optic Engineers Conference, OFC/NFOEC 2010, San Diego, CA, Mar. 21-25, pp. 1-20, 2010.

[45] P. Peng, J. Chen, and J. Sun, "Novel ring protection architecture for fiber sensor system," Japanese Journal of Applied Physics, vol. 50, no. 8, pp. 082501-1-082501-4, 2011.

[46] P. C. Peng, C. H. Chang, H. H. Lu, Y. T. Lin, J. W. Sun, and C. H. Jiang, "Novel optical add-drop multiplexer for wavelength-division-multiplexing networks," Optics Communications, vol. 285, no. 13-14, 15, pp. 3093-3099, 2012.

[47] D. N. Tibet-Shaban and N. J. Zhelezarski, "Design of a resilient optical fiber network for the multiplexing of sensors," M. S. thesis, Department of Electrical and Electronic Engineering, Public University of Navarra, Spain, 2009.

[48] P. Peng and S. Chi, "A reliable architecture for FBG sensor systems," Microwave and Optical Technology Letters, vol. 39, no. 6, 479-482, 2003.

[49] T. H. Wu, Fiber network service survivability. Norwood, MA: Artech House, 1992. 
[50] J. M. Senior, S. E. Moss, and S. D. Cusworth, "Multiplexing techniques for noninterferometric optical point-sensor networks: a review," Fiber and Integrated Optics, vol. 17, no. 1, pp. 3-20, 1998.

[51] K. T. V. Grattan and B. T. Meggitt, Optical fiber sensor technology. The Netherlands: Kluwer Academic Publishers, 2000.

[52] V. Montoya, M. Lopez-Amo, and S. Abad, "Improved double-fiber-bus with distributed optical amplification for wavelength-division multiplexing of photonic sensors," Photonics Technology Letters, vol. 12, no. 9, pp. 1270-1272, 2000.

[53] R. Hernandez-Lorenzo, M. Lopez-Amo, and
P. Urquhart, "Single and double distributed optical amplifier fiber bus networks with wavelength division multiplexing for photonic sensors," Journal of Lightwave Technology, vol. 16, no. 4, pp. 485-489, 1998.

[54] M. Schluter and P. Urquhart, "Optical fiber bus protection network to multiplex sensors: dedicated line and dedicated path operation," Journal of Lightwave Technology, vol. 29, no. 15, pp. 2204-2215, 2001.

[55] M. Fernandez-Vallejo and M. Lopez-Amo, "Optical fiber networks for remote fiber optic sensors," Sensors, vol. 12, no. 4, pp. 3929-3951, 2012. 